\title{
EFFECTIVE BOUNDS IN E.HOPF RIGIDITY FOR BILLIARDS AND GEODESIC FLOWS
}

\author{
MISHA BIALY
}

\begin{abstract}
In this paper we show that in some cases the E.Hopf rigidity phenomenon admits quantitative interpretation. More precisely we estimate from above the measure of the set $\mathcal{M}$ swept by minimal orbits. These estimates are sharp, i.e. if $\mathcal{M}$ occupies the whole phase space we recover the E.Hopf rigidity. We give these estimates in two cases: the first is the case of convex billiards in the plane, sphere or hyperbolic plane. The second is the case of conformally flat Riemannian metrics on a torus. It seems to be a challenging question to understand such a quantitative bounds for Burago-Ivanov theorem.
\end{abstract}

\section{IntRoduCtion AND THE RESUlT}

In this paper we estimate from above the measure of the set $\mathcal{M}$ in the phase space which is occupied by minimal orbits of a Hamiltonian system. These bounds are of obvious importance for dynamics because all "rotational" invariant torii, as well as Aubry-Mather sets are filled by minimal orbits.

The estimates provide the quantitative refinement of the E.Hopf rigidity. We prove these bounds for two Hamiltonian systems. The first system is a symplectic map of the cylinder corresponding to the billiard ball motion inside a convex curve $\gamma$ lying on a surface $\Sigma$ of constant curvature $0, \pm 1$. The second system is geodesic flow on a torus with conformally flat Riemannian metric.

Nowadays there are many cases and approaches where E.Hopf rigidity phenomenon is established. It is an important problem to understand which of them can be made quantitative. In particular, it seems to be a challenging question if it is possible to give a quantitative version for the Burago-Ivanov proof [5] of the E.Hopf conjecture.

Throughout the paper we denote by $\Omega$ the phase space of the Hamiltonian system in question. For the billiard in a convex domain bounded by closed curve $\gamma$, the phase space $\Omega$ is a cylinder: $\Omega=\gamma \times(-1,1)$ equipped with the standard symplectic form $d x \wedge d(\cos \varphi)$ giving the invariant measure $d \mu=\sin \varphi d x d \varphi$. Here and later the billiard map will be denoted by $T, x$ denotes arclength parameter on $\gamma$ and $\varphi$ is an inward angle. As for geodesic flow on the torus the phase space $\Omega$ is a unit tangent bundle $\Omega=T_{1} \mathbf{T}^{n}$ equipped with the Liouville measure.

Date: 20 April 2012.

2010 Mathematics Subject Classification. Primary:37J50;53C24.

Key words and phrases. Minimal geodesics, Minimal orbits, Convex Billiards, Conjugate points.

Partially supported by ISF grant 128/10. 
I shall use the following definition in this paper:

Definition 1.1. A geodesic will be called $m$-geodesic if it has no conjugate points. A billiard configuration $\left\{x_{n}\right\}$ will be called $m$-configuration if the second variation is negative definite between any two of end points. The corresponding orbits in the phase space will be called $m$-orbits.

Couple of remarks explain the definition. By Morse theory, for a geodesic to be without conjugate points is equivalent to have second variation positive definite between any two points. For billiards any discrete Jacobi field along every $m$-configuration vanishes not more than once and moreover change sign not more than once (see [1] and [8]).

We shall denote by $\mathcal{M} \subseteq \Omega$ the invariant subset of the phase space consisting of all $m$-orbits. Then it follows that $\mathcal{M}$ is a closed set (see [13 for the discrete case).

We shall introduce the notation for the portion of the phase space occupied by the set

$$
\Delta=\Omega \backslash \mathcal{M}, \delta=\mu(\Delta) / \mu(\Omega),
$$

where $\mu(\Omega)$ is the total measure of the phase space. Notice that the total measure equals $2 P$ for the case of billiards (here and later $P$ denotes the length of the boundary curve $\gamma$ and $A$ the area bounded by $\gamma$ ) and equals $\omega_{n-1} V o l_{g}\left(\mathbf{T}^{n}\right)$ for a Riemannian metric $g$ on the torus (here and below $\omega_{n-1}$ is the volume of the standard unit sphere $\mathbf{S}^{n-1} \subset \mathbf{R}^{n}$. So by the definition, $\delta \in[0,1]$ is dimensionless constant and the case $\delta=0$ is the case when all the orbits are $m$-orbits, which corresponds to the rigidity case. The purpose of this paper is to estimate $\delta$ from below.

We formulate first the bounds for the case of billiards:

Theorem 1.2. The following estimates hold true for a billiard table bounded by a simple closed strictly convex curve $\gamma$ on $\Sigma$ :

1. For the Euclidean plane, $\Sigma=\mathbf{R}^{2}$ :

$$
\delta \geq \frac{\pi\left(P^{2}-4 \pi A\right)}{4 P(P+\sqrt{4 \pi A})} \geq \frac{\pi\left(P^{2}-4 \pi A\right)}{8 P^{2}},
$$

and also

$$
\delta \geq \frac{\left(P^{2}-4 \pi A\right) k_{\min }}{8 P} .
$$

2. For the Hemisphere of, $\Sigma=\mathbf{S}^{2}$, for a curve $\gamma$ lying entirely in the hemisphere:

$$
\delta \geq \frac{\pi}{2 \arctan \left(\frac{1}{k_{\min }}\right)} \frac{P^{2}+A^{2}-4 \pi A}{P\left(2 \pi+\sqrt{P^{2}+(2 \pi-A)^{2}}\right)},
$$

3. For the Hyperbolic plane, $\Sigma=\mathbf{H}^{2}$, provided the boundary curve $\gamma$ is convex with respect to horocycles, that is $k_{\min }>1$ :

$$
\delta \geq \frac{\pi}{2 \operatorname{arctanh}\left(\frac{1}{\mathrm{k}_{\min }}\right)} \frac{P^{2}-A^{2}-4 \pi A}{P\left(2 \pi+\sqrt{(2 \pi+A)^{2}-P^{2}}\right)} .
$$

The following remarks are in order. 
Remark 1. Notice that the nominators of the bounds of the theorem contain the isoperimetric defect and therefore $\delta=0$ implies the curve $\gamma$ is a circle on $\Sigma$. Moreover, it follows from Bonnesen type inequalities (see [4]) that for small $\delta$ the curve is close to a circle in the sense Hausdorff distance.

I would also like to mention a somewhat related result of [9] where a quantitative version of a theorem by Mather is given estimating the area free from caustics inside the domain bounded by $\gamma$.

Remark 2. The estimate (1) uses the method of [1] where the Hopf rigidity for billiards was found. The bounds (2),(3), (4) on $\delta$ are obtained using the so called Mirror equation. The proof of E.Hopf rigidity for plane billiards using Mirror equation was obtained in [14] and later in [2] for the Sphere and Hyperbolic plane. Strangely the estimates (11) and (2) are incomparable, for some curves (1) is better and for others (2) is better. Let me mention that it remains unclear how to push the approach of [1] to work for Sphere and Hyperbolic plane.

Remark 3. Let me point out that in (4) for the $\mathbf{H}^{2}$ we need extra assumption on $\gamma$ to have $k_{\text {min }}>1$. For the case of rigidity when all the orbits are $m$-orbits this assumption is redundant as it is proved in [2]. However in general case it is not clear how to get rid of it.

Proof of Theorem 1.1 is given in Section 2,3.

Let me state now the result for geodesic flow. We consider Riemannian metric on the torus $\mathbf{T}^{n}=\mathbf{R}^{n} / \Gamma$ of the form $g=f g_{0}$ where $g_{0}$ is standard Euclidean metric on $\mathbf{R}^{n}$ and $f>0$ is a conformal factor. Hopf rigidity in this case was proved in [12] (and later in [6] by another method) generalizing the original proof of E.Hopf [11] and L.Green [10]. Our purpose is to make their approach quantitative and to estimate the Liouville measure $\delta$ from below. To do this one needs a refinement of the original Hopf method, because a straightforward application of the method does not lead to any estimate on $\delta$ (it is especially clear for the case $n=2$ ). For the proof I used below some of the earlier ideas of [3] on rigidity of Newton equations.

We shall split the result into two cases, $n=2$ and $n>2$.

Theorem 1.3. 1. For $n=2$, let $\psi: \mathbf{R}_{+} \rightarrow \mathbf{R}_{+}$be any positive smooth function. Denote by $\Psi(f)=\psi^{\prime}(f)\left(\frac{4}{f}-\frac{\psi^{\prime}(f)}{\psi(f)}\right)$. Then the following estimate holds true:

$$
\delta \geq \frac{\pi \int_{\mathbf{T}^{2}} \Psi(f)\left|\operatorname{grad}_{g_{0}} f\right|_{g_{0}}^{2} d \operatorname{Vol}_{g_{o}}}{4\|K\|_{C^{0}}\|\psi(f)\|_{C^{0}} \operatorname{Vol}\left(\mathbf{T}^{2}, g\right)}
$$

where $K$ is the curvature of the metric $g$.

2. For $n>2$, for any positive function $\psi: \mathbf{R}_{+} \rightarrow \mathbf{R}_{+}$introduce

$$
\Psi(f)=\Psi(f)=f^{\frac{n}{2}-1} \psi^{\prime}(f)\left(\frac{4}{f}-\frac{\psi^{\prime}(f)}{\psi(f)}\right)+(n-2) f^{\frac{n}{2}-3} \psi(f) .
$$

Then the following estimate holds

$$
\delta \geq \frac{(n-1) \omega_{n-1} \int_{\mathbf{T}^{n}} \Psi(f)\left|\operatorname{grad}_{g_{0}} f\right|_{g_{0}}^{2} d V \operatorname{Vol}_{g_{o}}}{4 n\|\operatorname{Ric}\|_{C^{0}}\|\psi(f)\|_{C^{0}} \operatorname{Vol}\left(\mathbf{T}^{n}, g\right)}
$$

where Ric stands for the Ricci tensor of $g$. 
Obviously this statement makes sense only if $\Psi$ is positive function. It turns out to be possible for many choices of $\psi$.

Corollary 1.4. For the particular choice of $\psi(f)=f^{\alpha}$ we have:

1. For $n=2$ and for every $\alpha$ in the range $0<\alpha<4$ it follows $\Psi(f)=\alpha(4-\alpha) f^{\alpha-2}$ and thus

$$
\delta \geq \frac{\pi \alpha(4-\alpha) \int_{\mathbf{T}^{2}} f^{\alpha-2}\left(f_{x_{1}}^{2}+f_{x_{2}}^{2}\right) d x_{1} d x_{2}}{4\|K\|_{C^{0}}\|f\|_{C^{0}}^{\alpha} \int f d x_{1} d x_{2}} .
$$

2. For $n>2$ and for every $\alpha$ in the range where $(n-2)+\alpha(4-\alpha)>0$ it follows

and thus

$$
\Psi(f)=((n-2)+\alpha(4-\alpha)) f^{\frac{n}{2}-3+\alpha}
$$

$$
\delta \geq((n-2)+\alpha(4-\alpha)) \frac{(n-1) \omega_{n-1} \int_{\mathbf{T}^{n}} f^{\frac{n}{2}-3+\alpha}\left|\operatorname{grad}_{g_{0}} f\right|_{g_{0}}^{2} d V o l_{g_{o}}}{4 n\|\operatorname{Ric}\|_{C^{0}}\left\|f^{\alpha}\right\|_{C^{0}} \operatorname{Vol}\left(\mathbf{T}^{n}, g\right)},
$$

Example 1. For $n=2$ and $\alpha=2$ one has

$$
\delta \geq \frac{\pi \int_{\mathbf{T}^{2}}\left(f_{x_{1}}^{2}+f_{x_{2}}^{2}\right) d x_{1} d x_{2}}{\|K\|_{C^{0}}\|f\|_{C^{0}}^{2} \int f d x_{1} d x_{2}}
$$

As for $n>2$ and $\alpha=2$ one has:

$$
\delta \geq \frac{(n+2)(n-1) \omega_{n-1} \int_{\mathbf{T}^{n}} f^{\frac{n}{2}-1}\left|\operatorname{grad}_{g_{0}} f\right|_{g_{0}}^{2} d V o l_{g_{o}}}{4 n\|\operatorname{Ric}\|_{C^{0}}\|f\|_{C^{0}}^{2} \operatorname{Vol}\left(\mathbf{T}^{n}, g\right)} .
$$

Remark 4. For both cases $n=2$ and $n>2$ and for $\alpha \geq 2$ one gets the strongest estimate in the Corollary for $\alpha=2$, because then the value of $\alpha(4-\alpha)$ becomes maximal. Analogously, for the case $n>2$ the estimate of the Corollary for $\alpha \leq 0$ is best possible for $\alpha=0$. Thus the meaningful range for $\alpha$ in the Corollary is $\alpha \in(0,2]$, for $n=2$ and $\alpha \in[0,2]$ for $n \geq 2$. Besides these remarks the estimates for different values of $\alpha$ seem to be incomparable. Let me also point out that unlike the case $n=2$, for $n>2$ the choice $\alpha=0$ is allowed and corresponds to the inequality considered by A.Knauf.

Proofs of Theorem 1.3 are given in Sections 4,5.

\section{ACKNOWLEDGEMENTS}

It is a pleasure to thank Semyon Alesker, Maxim Arnold, Victor Bangert and Wilderich Tuschmann for valuable discussions.

\section{EStimates FOR PLANAR BILLIARDS.}

It follows from [1] that along any m-configuration one can construct a positive discrete Jacobi field and then using this field to define a bounded measurable function $\omega: \mathcal{M} \rightarrow \mathbf{R}$, satisfying the inequality:

$$
\omega(y, \psi)-\omega(x, \varphi) \geq L_{11}(x, y)+2 L_{12}(x, y)+L_{22}(x, y) .
$$

Here and below $T:(x, \cos \varphi) \mapsto(y, \cos \psi) ; L$ denotes the distance between $\gamma(x)$ and $\gamma(y) ; x$ is an arclength parameter on $\gamma$ and subindexes of $L$ stand for partial derivatives with respect to $x, y$ respectively. 
Integrate against the invariant measure $\mu$ inequality (5) over the set $\mathcal{M}$ of all $m$-orbits. We get:

$$
0 \geq \int_{\mathcal{M}}\left(L_{11}(x, y)+2 L_{12}(x, y)+L_{22}(x, y)\right) d \mu .
$$

After a computation this leads to the inequality:

$$
\int_{\mathcal{M}} \frac{(\sin \varphi+\sin \psi)^{2}}{L} d \mu \leq \int_{\mathcal{M}}(k(x) \sin \varphi+k(y) \sin \psi) d \mu .
$$

The LHS of (6) can be estimated from below by Cauchy-Schwartz and Santalo formulas:

$$
L H S \geq \frac{\left(\int_{\mathcal{M}}(\sin \varphi+\sin \psi) d \mu\right)^{2}}{\int_{\mathcal{M}} L d \mu} \geq \frac{\left(2 \int_{\mathcal{M}} \sin \varphi d \mu\right)^{2}}{\int_{\Omega} L d \mu}=\frac{\left(2 \int_{\mathcal{M}} \sin \varphi d \mu\right)^{2}}{2 \pi A} .
$$

The RHS of (6) can be estimated:

$$
R H S=2 \int_{\mathcal{M}} k(x) \sin \varphi d \mu \leq 2 \int_{\Omega} k(x) \sin \varphi d \mu=2 \pi^{2} .
$$

Therefore (6) gives the following

$$
\frac{2 \int_{\mathcal{M}} \sin \varphi d \mu}{\sqrt{2 \pi A}} \leq \sqrt{2 \pi^{2}}
$$

Therefore

$$
2 \int_{\mathcal{M}} \sin \varphi d \mu \leq \pi \sqrt{4 \pi A}
$$

Estimating the left hand side of the last inequality we get

$$
\pi P-4 \delta P \leq 2 \int_{\Omega} \sin \varphi d \mu-2 \int_{\Delta} \sin \varphi d \mu=2 \int_{\mathcal{M}} \sin \varphi d \mu \leq \pi \sqrt{4 \pi A} .
$$

Thus

so that

$$
\frac{\sqrt{4 \pi A}}{P} \geq 1-\frac{4}{\pi} \delta
$$

$$
\frac{\pi}{4}\left(1-\frac{\sqrt{4 \pi A}}{P}\right) \leq \delta .
$$

Then

$$
\frac{\pi}{4}\left(\frac{P^{2}-4 \pi A}{P(2 P)}\right) \leq \frac{\pi}{4}\left(\frac{P^{2}-4 \pi A}{P(P+\sqrt{4 \pi A})}\right) \leq \delta .
$$

This proves (1).

In order to prove (2) we use another measurable function defined on the subset filled by $m$-orbits

$$
a: \mathcal{M} \rightarrow \mathbf{R}, 0<a(x, \varphi)<L(x, \varphi),
$$

which is related in fact to the function $\omega$ discussed in the proof of (11) (see [2]). This function satisfies the Mirror equation for any point $(x, \varphi) \in \mathcal{M}$ :

$$
\frac{1}{a(x, \varphi)}+\frac{1}{L\left(T^{-1}(x, \varphi)\right)-a\left(T^{-1}(x, \varphi)\right)}=\frac{2 k(x)}{\sin \varphi},
$$

Then it follows

$$
\frac{a(x, \varphi)+\left(L\left(T^{-1}(x, \varphi)\right)-a\left(T^{-1}(x, \varphi)\right)\right.}{2} \geq \frac{\sin \varphi}{k(x)} .
$$


Integrate this inequality against the invariant measure $d \mu$ over the set $\mathcal{M}$. We have:

$$
\frac{1}{2} \int_{\mathcal{M}} L d \mu \geq \int_{\mathcal{M}} \frac{\sin \varphi}{k(x)}
$$

The LHS of (8) can be estimated using Santalo formula:

$$
\pi A=\frac{1}{2} \int_{\Omega} L d \mu \geq \frac{1}{2} \int_{\mathcal{M}} L d \mu .
$$

And for the RHS we have using Cauchy-Schwartz

$$
\begin{aligned}
& \int_{\mathcal{M}} \frac{\sin \varphi}{k(x)}=\int_{\Omega} \frac{\sin \varphi}{k(x)}-\int_{\Delta} \frac{\sin \varphi}{k(x)} \geq \\
\geq & \frac{\pi}{2} \int_{0}^{P} \frac{1}{k(x)} d x-\frac{2 \delta P}{k_{\min }} \geq \frac{\pi}{2} \cdot \frac{P^{2}}{2 \pi}-\frac{2 \delta P}{k_{\min }} .
\end{aligned}
$$

Therefore (8) yields:

$$
\pi A \geq \frac{P^{2}}{4}-\frac{2 \delta P}{k_{\min }}
$$

which is equivalent to (2). This completes the proof of (2).

\section{Billiard on the Sphere and the Hyperbolic plane}

The Mirror equation for billiards on Hemisphere and Hyperbolic plane is obtained in [2]. For the Hemisphere, there exists a measurable function

$$
a: \mathcal{M} \rightarrow \mathbf{R}, 0<a(x, \varphi)<L(x, \varphi)
$$

such that for any point $(x, \varphi) \in \mathcal{M}$ the following holds:

$$
\cot (a(x, \varphi))+\cot \left(L\left(T^{-1}(x, \varphi)\right)-a\left(T^{-1}(x, \varphi)\right)\right)=\frac{2 k(x)}{\sin \varphi} .
$$

It implies:

$$
\cot \frac{a(x, \varphi)+L\left(T^{-1}(x, \varphi)\right)-a\left(T^{-1}(x, \varphi)\right)}{2} \leq \frac{k(x)}{\sin \varphi},
$$

Equivalently

$$
\frac{a(x, \varphi)+L\left(T^{-1}(x, \varphi)\right)-a\left(T^{-1}(x, \varphi)\right)}{2} \geq \arctan \left(\frac{\sin \varphi}{k(x)}\right) .
$$

Integrating over $\mathcal{M}$ with respect to the invariant measure $d \mu=\sin \varphi d x d \varphi$ we get:

$$
\int_{\mathcal{M}} L d \mu \geq 2 \int_{\mathcal{M}} \arctan \left(\frac{\sin \varphi}{k(x)}\right) d \mu
$$

For the LHS of (10) we have:

$$
\int_{\mathcal{M}} L d \mu \leq \int_{\Omega} L d \mu=2 \pi A .
$$

As for the RHS of (10) we compute and use Gauss-Bonnet to get:

$2 \int_{\mathcal{M}} \arctan \left(\frac{\sin \varphi}{k(x)}\right) d \mu=2 \int_{\Omega} \arctan \left(\frac{\sin \varphi}{k(x)}\right) d \mu-2 \int_{\Delta} \arctan \left(\frac{\sin \varphi}{k(x)}\right) d \mu$ 


$$
\begin{aligned}
& \geq 2 \int_{0}^{P} d x \int_{0}^{\pi} \arctan \left(\frac{\sin \varphi}{k(x)}\right) \sin \varphi d \varphi-4 P \delta \arctan \left(\frac{1}{k_{\min }}\right)= \\
& \quad=2 \pi \int_{0}^{P}\left(\sqrt{k^{2}(x)+1}-k(x)\right) d x-4 P \delta \arctan \left(\frac{1}{k_{\min }}\right)= \\
& =2 \pi \int_{0}^{P} \sqrt{k^{2}(x)+1} d x-2 \pi(2 \pi-A)-4 P \delta \arctan \left(\frac{1}{k_{\text {min }}}\right) .
\end{aligned}
$$

Substitute now the estimates back into (10):

$$
\int_{0}^{P} \sqrt{k^{2}(x)+1} d x \leq 2 \pi+\frac{2 P \delta}{\pi} \arctan \left(\frac{1}{k_{\min }}\right) .
$$

But then the following two inequalities follow. The first one:

$$
\int_{0}^{P}\left(\sqrt{k^{2}(x)+1}-1\right) d x \leq 2 \pi-P+\frac{2 P \delta}{\pi} \arctan \left(\frac{1}{k_{\min }}\right) .
$$

And the second is:

$$
\int_{0}^{P}\left(\sqrt{k^{2}(x)+1}+1\right) d x \leq 2 \pi+P+\frac{2 P \delta}{\pi} \arctan \left(\frac{1}{k_{\min }}\right) .
$$

Multiplying two of them and using Cauchy-Schwartz we get:

$$
(2 \pi-A)^{2}=\left(\int_{0}^{P} k(x) d x\right)^{2} \leq\left(2 \pi+\frac{2 P \delta}{\pi} \arctan \left(\frac{1}{k_{\min }}\right)\right)^{2}-P^{2} .
$$

Therefore

$$
\sqrt{P^{2}+(2 \pi-A)^{2}} \leq 2 \pi+\frac{2 P \delta}{\pi} \arctan \left(\frac{1}{k_{\min }}\right)
$$

Thus

$$
\begin{gathered}
\frac{P^{2}-4 \pi A+A^{2}}{\sqrt{P^{2}+(2 \pi-A)^{2}}+2 \pi}=\sqrt{P^{2}+(2 \pi-A)^{2}}-2 \pi \leq \\
\leq \frac{2 P \delta}{\pi} \arctan \left(\frac{1}{k_{\min }}\right) .
\end{gathered}
$$

But this is exactly (3), so the proof for the Hemisphere is finished.

For the Hyperbolic plane the proof is similar. Let me sketch the main steps. Let me remind first that for the Hyperbolic case we need an additional requirement $k(x) \geq k_{\min }>1$. In particular this implies

$$
P<k_{\min } P \leq \int_{0}^{P} k(x) d x=2 \pi+A .
$$

We start again with a measurable function

$$
a: \mathcal{M} \rightarrow \mathbf{R}, 0<a(x, \varphi)<L(x, \varphi)
$$

such that for any point $(x, \varphi) \in \mathcal{M}$ the Mirror equation holds:

$$
\operatorname{coth}(a(x, \varphi))+\operatorname{coth}\left(L\left(T^{-1}(x, \varphi)\right)-a\left(T^{-1}(x, \varphi)\right)\right)=\frac{2 k(x)}{\sin \varphi} .
$$

This leads to the inequality:

$$
a(x, \varphi)+L\left(T^{-1}(x, \varphi)\right)-a\left(T^{-1}(x, \varphi)\right) \geq 2 \operatorname{arctanh}\left(\frac{\sin \varphi}{\mathrm{k}(\mathrm{x})}\right) .
$$


Integrating over $\mathcal{M}$ we get

$$
\int_{\mathcal{M}} L d \mu \geq 2 \int_{\mathcal{M}} \operatorname{arctanh}\left(\frac{\sin \varphi}{\mathrm{k}(\mathrm{x})}\right) \mathrm{d} \mu
$$

which leads to the inequality:

$$
2 \pi A \geq 2 \int_{\mathcal{M}} L d \mu \geq \pi \int_{0}^{P}\left(k(x)-\sqrt{k^{2}(x)-1}\right) d x-4 P \delta \operatorname{arctanh}\left(\frac{1}{\mathrm{k}_{\min }}\right) .
$$

This implies by Gauss-Bonnet:

$$
\int_{0}^{P} \sqrt{k^{2}(x)-1} d x \geq 2 \pi-\frac{2 P \delta}{\pi} \operatorname{arctanh}\left(\frac{1}{\mathrm{k}_{\min }}\right) .
$$

By Cauchy Schwartz we have;

$\int_{0}^{P} \sqrt{k^{2}(x)-1} d x \leq\left(\int_{0}^{P}(k(x)-1) d x \int_{0}^{P}(k(x)+1) d x\right)^{\frac{1}{2}}=\sqrt{(A+2 \pi)^{2}-P^{2}}$.

Thus we get:

$$
\sqrt{(A+2 \pi)^{2}-P^{2}} \geq 2 \pi-\frac{2 P \delta}{\pi} \operatorname{arctanh}\left(\frac{1}{\mathrm{k}_{\min }}\right) .
$$

This completes the proof.

\section{Proof of the estimates for Geodesic flows in $n=2$.}

The original E.Hopf method needs a modification in order to get bounds on the measure $m$-geodesics. This goes as follows.

First, following E.Hopf, for every geodesic with no conjugate points one constructs by a limiting procedure a positive solution of the Jacobi equation and then a measurable bounded function $\omega: \mathcal{M} \rightarrow \mathbf{R}$ which is smooth along the orbits of the geodesic flow satisfying the Riccati equation:

$$
\dot{\omega}+\omega^{2}+K=0 .
$$

Here the derivative is taken in the direction of the vector of the geodesic flow in $T_{1} \mathbf{T}^{2}$, and $K$ is the curvature of the conformal metric $g=f\left(d x_{1}^{2}+d x_{2}^{2}\right)$. Let me remind that $\mathcal{M}$ is a closed subset of the phase space $\Omega=T_{1} \mathbf{T}^{2}$ invariant under the geodesic flow.

Multiplying both sides of the equation by a positive factor $\psi(f)$ we get:

$$
\frac{d}{d t}(\psi \omega)-\omega \frac{d}{d t}(\psi(f))+\psi(f) \omega^{2}+\psi(f) K=0,
$$

Which leads to

$$
\frac{d}{d t}(\psi(f) \omega)-\psi^{\prime}(f)\left(f_{x_{1}} \dot{x_{1}}+f_{x_{2}} \dot{x_{2}}\right) \omega+\psi(f) \omega^{2}+\psi(f) K=0,
$$

For $T_{1} \mathbf{T}^{2}$ we have $\dot{x_{1}}=\frac{1}{\sqrt{f}} \cos \varphi, \dot{x_{2}}=\frac{1}{\sqrt{f}} \sin \varphi$ therefore

$$
\frac{d}{d t}(\psi(f) \omega)-\psi^{\prime}(f)\left(\frac{f_{x_{1}}}{\sqrt{f}} \cos \varphi+\frac{f_{x_{2}}}{\sqrt{f}} \sin \varphi\right) \omega+\psi(f) \omega^{2}+\psi(f) K=0
$$

Integrate the last equation over the set $\mathcal{M}$ against the Liouville measure $d \mu=f d x_{1} d x_{2} d \varphi$ and use its invariance under the geodesic flow to get 
(16) $-\int_{\mathcal{M}} \psi^{\prime}\left(\frac{f_{x_{1}}}{\sqrt{f}} \cos \varphi+\frac{f_{x_{2}}}{\sqrt{f}} \sin \varphi\right) \omega d \mu+\int_{\mathcal{M}} \psi \omega^{2} d \mu+\int_{\mathcal{M}} \psi K d \mu=0$,

Denote the first and the last term in equation (16) by $A$ and $C$ respectively. Then, by Cauchy-Schwartz for $A$ we have:

$$
\begin{aligned}
A \geq & -\left(\int_{\mathcal{M}} \frac{\left(\psi^{\prime}\right)^{2}}{f \psi}\left(f_{x_{1}} \cos \varphi+f_{x_{2}} \sin \varphi\right)^{2} d \mu\right)^{\frac{1}{2}}\left(\int_{\mathcal{M}} \psi \omega^{2} d \mu\right)^{\frac{1}{2}} \geq \\
- & \left(\int_{\Omega} \frac{\left(\psi^{\prime}\right)^{2}}{f \psi}\left(f_{x_{1}} \cos \varphi+f_{x_{2}} \sin \varphi\right)^{2} d \mu\right)^{\frac{1}{2}}\left(\int_{\mathcal{M}} \psi \omega^{2} d \mu\right)^{\frac{1}{2}}= \\
& -\left(\pi \int_{\mathbf{T}^{2}} \frac{\left(\psi^{\prime}\right)^{2}}{\psi}\left(f_{x_{1}}^{2}+f_{x_{2}}^{2}\right) d x_{1} d x_{2}\right)^{\frac{1}{2}}\left(\int_{\mathcal{M}} \psi \omega^{2} d \mu\right)^{\frac{1}{2}}
\end{aligned}
$$

The third term $C$ can be written as follows:

$$
\begin{aligned}
C & =\int_{\Omega} \psi K d \mu-\int_{\Delta} \psi K d \mu \geq \int_{\Omega} \psi K d \mu-\|\psi(f)\|_{C_{0}}\|K\|_{C_{0}} \mu(\Delta)= \\
& =2 \pi \int_{\mathbf{T}^{2}} \psi(f) K f d x_{1} d x_{2}-\|\psi(f)\|_{C_{0}}\|K\|_{C_{0}} \cdot \delta \cdot \operatorname{Vol}\left(\mathbf{T}^{2}, g\right) .
\end{aligned}
$$

Substitute the explicit expression for $K=-\frac{\Delta(\log f)}{2 f}$ and integrate by parts to get:

$$
C \geq \pi \int_{\mathbf{T}^{2}} \frac{\psi^{\prime}(f)}{f}\left(f_{x_{1}}^{2}+f_{x_{2}}^{2}\right) d x_{1} d x_{2}-\|\psi(f)\|_{C_{0}}\|K\|_{C_{0}} \delta \cdot \operatorname{Vol}\left(\mathbf{T}^{2}, g\right) .
$$

Use the estimates of the terms $A$ and $C$ in the equation (16)

$$
-\left(\pi \int_{\mathbf{T}^{2}} \frac{\left(\psi^{\prime}\right)^{2}}{\psi}\left(f_{x_{1}}^{2}+f_{x_{2}}^{2}\right) d x_{1} d x_{2}\right)^{\frac{1}{2}} \cdot X+X^{2}+
$$

(17) $+\pi \int_{\mathbf{T}^{2}} \frac{\psi^{\prime}(f)}{f}\left(f_{x_{1}}^{2}+f_{x_{2}}^{2}\right) d x_{1} d x_{2}-\|\psi(f)\|_{C_{0}}\|K\|_{C_{0}} \cdot \delta \cdot \operatorname{Vol}\left(\mathbf{T}^{2}, g\right) \leq 0$,

where we denoted by

$$
X=\left(\int_{\mathcal{M}} \psi \omega^{2} d \mu\right)^{\frac{1}{2}}
$$

Next notice that (17) is a quadratic inequality in $X$ and therefore the discriminant must be non-negative:

$$
\begin{gathered}
\pi \int_{\mathbf{T}^{2}} \frac{\left(\psi^{\prime}\right)^{2}}{\psi}\left(f_{x_{1}}^{2}+f_{x_{2}}^{2}\right) d x_{1} d x_{2}-4 \pi \int_{\mathbf{T}^{2}} \frac{\psi^{\prime}(f)}{f}\left(f_{x_{1}}^{2}+f_{x_{2}}^{2}\right) d x_{1} d x_{2}+ \\
+4\|\psi(f)\|_{C_{0}}\|K\|_{C_{0}} \cdot \delta \cdot \operatorname{Vol}\left(\mathbf{T}^{2}, g\right) \geq 0 .
\end{gathered}
$$

But this is precisely the inequality which is claimed. This completes the proof for $n=2$. 
5. Proof of the estimates for Geodesic flows in $n>2$.

In this case we modify the approach by L.Green and A.Knauf in a similar way that we did for the case $n=2$. We start with a measurable bounded function (see [10] or [7] for the construction) $\omega: \mathcal{M} \rightarrow \mathbf{R}$ which satisfies the differential inequality:

$$
\frac{d}{d t} \omega+\frac{\omega^{2}}{n-1}+R \leq 0,
$$

where the derivative is along the geodesic flow and $R$ is a function

$$
R: \Omega \rightarrow \mathbf{R}, R(v)=\operatorname{Ric}(v, v) .
$$

Multiplying both sides of the inequality by a positive factor $\psi(f)$ we get:

$$
\frac{d}{d t}(\psi \omega)-\psi^{\prime}(f) \dot{f} \omega+\psi(f) \frac{\omega^{2}}{n-1}+\psi(f) R \leq 0,
$$

Integrate against the invariant measure $d \mu=f^{\frac{n}{2}} d x d o$ over the set $\mathcal{M} \subseteq \Omega$ (where $d x, d o$ are the standard measures on Euclidean space and on the unit sphere).

$$
-\int_{\mathcal{M}} \psi^{\prime}(f) \dot{f} \omega d \mu+\int_{\mathcal{M}} \frac{\psi \omega^{2}}{n-1} d \mu+\int_{\mathcal{M}} \psi R d \mu \leq 0
$$

We can estimate the first term $A$ and the last $C$ as follows. By CauchySchwartz inequality

$$
\begin{gathered}
A \geq-\left(\int_{\mathcal{M}} \frac{\left(\psi^{\prime}\right)^{2}}{\psi}(\dot{f})^{2} d \mu\right)^{\frac{1}{2}}\left(\int_{\mathcal{M}} \psi \omega^{2} d \mu\right)^{\frac{1}{2}} \geq \\
-\left(\int_{\Omega} \frac{\left(\psi^{\prime}\right)^{2}}{\psi}(\dot{f})^{2} d \mu\right)^{\frac{1}{2}}\left(\int_{\mathcal{M}} \psi \omega^{2} d \mu\right)^{\frac{1}{2}}= \\
-\left(\int_{\Omega} \frac{\left(\psi^{\prime}\right)^{2}}{f \psi}<\operatorname{grad}_{g_{0}} f, f \dot{x}>_{g_{0}}^{2} f^{\frac{n}{2}} d x d o\right)^{\frac{1}{2}}\left(\int_{\mathcal{M}} \psi \omega^{2} d \mu\right)^{\frac{1}{2}}= \\
-\left(\frac{\omega_{n-1}}{n} \int_{\mathbf{T}^{n}} \frac{\left(\psi^{\prime}\right)^{2}}{\psi}\left\|\operatorname{grad}_{g_{0}} f\right\|_{g_{0}}^{2} f^{\frac{n}{2}-1} d x\right)^{\frac{1}{2}}\left(\int_{\mathcal{M}} \psi \omega^{2} d \mu\right)^{\frac{1}{2}} .
\end{gathered}
$$

For the last term $C$ we have:

$$
\begin{aligned}
& C=\int_{\Omega} \psi R d \mu-\int_{\Delta} \psi R d \mu \geq \int_{\Omega} \psi R d \mu-\|\psi(f)\|_{C_{0}}\|R\|_{C_{0}} \mu(\Delta)= \\
& =\frac{\omega_{n-1}}{n} \int_{\mathbf{T}^{n}} \psi(f) \operatorname{Scal}(g) f^{\frac{n}{2}} d x-\|\psi(f)\|_{C_{0}}\|R\|_{C_{0}} \cdot \delta \cdot \operatorname{Vol}\left(\mathbf{T}^{n}, g\right),
\end{aligned}
$$

where $\operatorname{Scal}(g)$ is the Scalar curvature of $g$. Substitute the explicit expression for Scal,

$$
\operatorname{Scal}(g)=(1-n) f^{-2} \Delta f+\frac{(1-n)(n-6)}{4} f^{-3}\left\|\operatorname{grad}_{g_{0}} f\right\|_{g_{0}}^{2}
$$

and integrate by parts the term with the Laplacian to get:

$$
C \geq \frac{\omega_{n-1}(1-n)}{n}\left(\int_{\mathbf{T}^{n}} \psi(f) f^{\frac{n}{2}-2} \Delta f d x+\int_{\mathbf{T}^{n}} \frac{(n-6)}{4} \psi(f) f^{\frac{n}{2}-3}\left\|\operatorname{grad}_{g_{0}} f\right\|_{g_{0}}^{2} d x\right)-
$$




$$
\begin{gathered}
-\|\psi(f)\|_{C_{0}}\|R\|_{C_{0}} \cdot \delta \cdot \operatorname{Vol}\left(\mathbf{T}^{n}, g\right) \geq \\
\geq \frac{\omega_{n-1}(1-n)}{n} \int_{\mathbf{T}^{n}}\left(-\left(\psi(f) f^{\frac{n}{2}-2}\right)^{\prime}+\frac{(n-6)}{4} \psi(f) f^{\frac{n}{2}-3}\right)\left\|\operatorname{grad}_{g_{0}} f\right\|_{g_{0}}^{2} d x- \\
-\|\psi(f)\|_{C_{0}}\|R\|_{C_{0}} \cdot \delta \cdot \operatorname{Vol}\left(\mathbf{T}^{n}, g\right)=: \tilde{C} .
\end{gathered}
$$

Substituting the estimates on $A, C$ and the notation $X=\left(\int_{\mathcal{M}} \psi(f) \omega^{2} d \mu\right)^{\frac{1}{2}}$ into (19) we get the quadratic inequality:

$$
-X \cdot\left(\frac{\omega_{n-1}}{n} \int_{\mathbf{T}^{n}} \frac{\left(\psi^{\prime}\right)^{2}}{\psi}\left\|\operatorname{grad}_{g_{0}} f\right\|_{g_{0}}^{2} f^{\frac{n}{2}-1} d x\right)^{\frac{1}{2}}+\frac{1}{n-1} X^{2}+\tilde{C} \leq 0 .
$$

So the discriminant $D$ must be non-negative, which leads to

Then

$$
\frac{\omega_{n-1}}{n} \int_{\mathbf{T}^{n}} \frac{\left(\psi^{\prime}\right)^{2}}{\psi}\left\|\operatorname{grad}_{g_{0}} f\right\|_{g_{0}}^{2} f^{\frac{n}{2}-1} d x-\frac{4}{n-1} \tilde{C} \geq 0
$$

$$
\begin{gathered}
\frac{4}{n-1}\|\psi(f)\|_{C_{0}}\|R\|_{C_{0}} \cdot \delta \cdot \operatorname{Vol}\left(\mathbf{T}^{n}, g\right) \geq \\
-\frac{\omega_{n-1}}{n} \int_{\mathbf{T}^{n}}\left(\frac{\left(\psi^{\prime}\right)^{2}}{\psi} f^{\frac{n}{2}-1}-4\left(\psi(f) f^{\frac{n}{2}-2}\right)^{\prime}+(n-6) \psi(f) f^{\frac{n}{2}-3}\right)\left\|g r a d_{g_{0}} f\right\|_{g_{0}}^{2} d x .
\end{gathered}
$$

By the definition of $\Psi(f)$ this is equivalent to:

$$
\frac{4}{n-1}\|\psi(f)\|_{C_{0}}\|R\|_{C_{0}} \cdot \delta \cdot \operatorname{Vol}\left(\mathbf{T}^{n}, g\right) \geq \frac{\omega_{n-1}}{n} \int_{\mathbf{T}^{n}} \Psi(f)\left\|\operatorname{grad}_{g_{0}} f\right\|_{g_{0}}^{2} d x
$$

This proves the claim for $n>2$.

\section{REFERENCES}

[1] Bialy, M. Convex billiards and a theorem by E. Hopf. Math. Z. 214 (1993), no. 1, $147-154$.

[2] Bialy, M. Hopf rigidity for convex billiards on the hemisphere and hyperbolic plane. Discrete Contin. Dyn. Syst. 33 (2013), no. 9, 3903-3913.

[3] Bialy, M.; Polterovich, L. Hopf-type rigidity for Newton equations. Math. Res. Lett. 2 (1995), no. 6, 695-700.

[4] Burago, Yu.; Zalgaller, V. Geometric inequalities. (Translated by A. B. Sossinsky), Springer-Verlag, Berlin, Heidelberg, New York, London, Paris, Tokyo, 1988.

[5] Burago, D.; Ivanov, S. Riemannian tori without conjugate points are flat. Geom. Funct. Anal. 4 (1994), no. 3, 259-269.

[6] Croke, C.; Fathi, A. An inequality between energy and intersection. Bull. London Math. Soc. 22 (1990), no. 5, 489-494.

[7] Chavel, I. Riemannian geometry-a modern introduction. Cambridge Tracts in Mathematics, 108. Cambridge University Press, Cambridge, 1993.

[8] Elaydi, S. An introduction to difference equations. Undergraduate Texts in Mathematics. Springer, New York, 2005.

[9] Gutkin, E.; Katok, A. Caustics for inner and outer billiards. Comm. Math. Phys. 173 (1995), no. 1, 101-133.

[10] Green, L. W. A theorem of E. Hopf. Michigan Math. J. 519583134.

[11] Hopf, E. Closed surfaces without conjugate points. Proc. Nat. Acad. Sci. U. S. A. 34, (1948). 47-51.

[12] Knauf, A. Closed orbits and converse KAM theory. Nonlinearity 3 (1990), no. 3, 961-973.

[13] MacKay,R.,Meiss,J., Strark,J. Converse KAM theory for symplectic twist maps, Nonlinearity 2 (1989) 555-570. 
[14] Wojtkowski, M. Two applications of Jacobi fields to the billiard ball problem. J. Differential Geom. 40 (1994), no. 1, 155-164.

School of Mathematical Sciences, Raymond and Beverly Sackler Faculty of Exact Sciences, Tel Aviv University, Israel

E-mail address: bialy@post.tau.ac.il 